\title{
Rare earth doped III-nitrides for optoelectronics
}

\author{
K.P. O'Donnell ${ }^{\mathrm{a}}$ and B. Hourahine \\ SUPA Department of Physics, University of Strathclyde, Glasgow G4 0NG, Scotland, UK
}

Received: 18 August 2006 / Accepted: 1 September 2006

Published online: 28 October 2006 - (C) EDP Sciences

\begin{abstract}
Rare-earth (RE) doped III-nitrides, prepared by in-situ doping during growth or by ion implantation and annealing, are promising materials for visible light emitting displays. In addition, they are extremely challenging theoretically, on account of the complexity of the sharp inter- $4 f$ optical transitions, which are allowed only through the mixing by non-centrosymmetric crystal fields of the inner $4 f$ orbitals with higher-lying states of opposite parity. We review recent experimental and theoretical work on Er-, Eu- and Tm-doped III-nitride compounds and alloys which has been carried out with a view to establishing the lattice location of RE in these materials and the probable nanostructure of the centres which are responsible for their luminescence. The isolated site $\mathrm{RE}_{\mathrm{III}}$ is found to be both optically and electrically inactive, but in association with neighbouring intrinsic defects (most probably nitrogen vacancies) RE can generate a small family of similar optically active sites. Such a family is held to be responsible for the site multiplicity that is a common feature of the spectroscopy of RE-doped III-nitrides.
\end{abstract}

PACS. $61.72 . \mathrm{Vv}$ Doping and impurity implantation in III-V and II-VI semiconductors - 78.66.Fd III-V semiconductors - 71.55.Eq III-V semiconductors

\section{Introduction}

Rare Earth (RE) doping offers an all-nitride route to the realisation of optoelectronic devices that emit visible light [1]. For example, gallium nitride $(\mathrm{GaN})$, doped with thulium (Tm), erbium (Er) and europium (Eu) will, when excited by a laser or electron beam, emit spectrally pure light in a few narrow bands in the blue, green and red regions, respectively. The RE emissions may be additively mixed to stimulate most of the responses possible in human colour vision. In contrast, it is well known that the emission efficiency of the commercially successful indium gallium nitride alloy, $\operatorname{In}_{x} \mathrm{Ga}_{1-x} \mathrm{~N}$, decreases dramatically as the wavelength increases above, say, $550 \mathrm{~nm}$, corresponding to the green visual peak [2]. The decrease in efficiency with increasing wavelength is well illustrated by comparing maps of composition and cathodoluminescence (CL) intensity of a graded InGaN epilayer, as shown in Figure 1.

Wavelength tuning of emission from semiconductor alloys (solid solutions) is easily achieved by changing the composition. Practically, in III-nitride epitaxy, this will require a change in the growth temperature of the sample, which may have a collateral effect on its crystal quality. A shift in peak wavelength from $570 \mathrm{~nm}$ to $700 \mathrm{~nm}$, corresponding to an increase of InN fraction from $x \sim 0.25$ to 0.4 , is accompanied by a decrease of more than an order of magnitude in the photoluminescence (PL) efficiency of

\footnotetext{
a e-mail: k.p.odonnell@strath.ac.uk
}

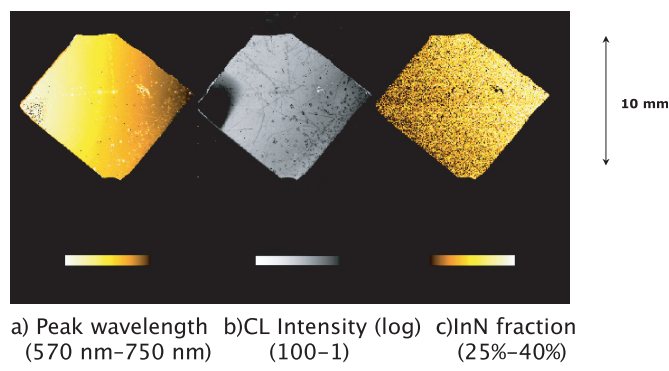

Fig. 1. An $\operatorname{In}_{x} \mathrm{Ga}_{1-x} \mathrm{~N}$ sample with a lateral gradation in composition illustrates the relatively poor performance of this alloy as a red light emitter. The wavelength peak (a) of the emission shifts from $570 \mathrm{~nm}$ to $750 \mathrm{~nm}$ and its intensity (b) fades by two orders of magnitude as the InN fraction (c) increases from 0.25 to 0.40 . Images were collected by an electron probe microanalyser adapted for CL spectral imaging.

$\mathrm{In}_{x} \mathrm{Ga}_{1-x} \mathrm{~N}$ at room temperature. As far as we are aware, no comparable study has been carried out for those infrared (IR) emitting samples with $\mathrm{InN}$ fraction higher than $x=0.4$ that are now becoming more widely available. At very low values of $x$, however, the PL and electroluminescence (EL) intensities are found to increase with InN content [3]. Hence, $\operatorname{In}_{x} \mathrm{Ga}_{1-x} \mathrm{~N}$ devices peak in emission efficiency near $450 \mathrm{~nm}$ and show a severe falling off towards the red end of the visible spectrum. Correspondingly, most of the many thousands of reports published on InGaN luminescence in the last 10 years deal exclusively with blue-light emitting samples. 
The study of luminescence from RE ions embedded in semiconductors has quite a long history; of particular interest is the $1.54 \mu \mathrm{m}$ IR band which is emitted by $\mathrm{Er}^{3+}$ in a large number of hosts [4]; this band is suitable for transmission through silica fibre over long distances. Quite early on, Favennec et al. established the principle [5] that semiconductors with wider band gaps suffer less from the temperature quenching of the luminescence that is expected in bulk hosts. The exploration of wide gap III-nitrides as RE hosts followed the development of suitable epitaxial growth techniques, notably Molecular Beam Epitaxy (MBE) and Metallorganic Vapour Phase Epitaxy (MOVPE), in the 1990's. Steckl and co-workers have provided a recent comprehensive review of the application of RE-doped MBE-grown nitrides to EL devices [6]. In the last few years, the successful incorporation of RE ions in nitride nanostructures, again using MBE, has been shown to offer considerable promise for future developments in the field [7].

Luminescence from RE ions in solid hosts involves transitions within the $4 f$ electronic shell that are strictly forbidden in the free ion by the Laporte selection rule but become more or less allowed through the admixture of states of opposite parity induced by the crystal field in solids. It is therefore of some importance to establish the lattice location of RE ions in nitrides since this will primarily determine the symmetry of the local environment. The totality of the environment, insofar as it influences the optical properties of a particular RE ion, shall be referred to in this review as a 'site'. The unperturbed site, RE $\mathrm{EII}_{\mathrm{I}}$ denoting $\mathrm{RE}^{3+}$ substituting for the group-III species, say $\mathrm{Ga}$ in semi-ionic GaN, is probable on the basis of charge equivalence of the ions, but might not be the majority one. We will reserve the term 'defect' for intrinsic lattice disorder, vacancies, self-interstitials, etc., which are not actively luminescent, but may influence RE luminescence for better or worse. An actively luminescent site is sometimes called a luminescence 'centre'.

Given a multiplicity of sites, it may be possible to engineer the occupation statistics of available sites in such a way as to maximise the RE emission intensity. We include in this design approach the idea of enhancing the luminescence efficiency by the addition of co-dopants, which may act either locally, to influence the site symmetry, or non-locally, as 'sensitisers' of the luminescence, and the use of various physical treatments (thermal annealing, irradiation, etc.) to enhance the luminescence output. At the same time, we should be aware that the transfer of excitation from the band states of a semiconductor host to the inner electronic shells of RE ions, perhaps involving the mediation of defects and/or the migration and localisation of excitons, is itself not a well-understood process; this process may well favour the excitation of particular sites through some as yet undiscovered physical mechanism. The defect engineering approach to optimising III$\mathrm{N}: \mathrm{RE}$ light emitters asks this question first of all: is RE emission due mainly to: (a) a majority site (with a low oscillator strength) or (b) a minority site of exceptionally high luminescence efficiency (a 'magic' site).
Most of the work to be presented in this topical review was carried out by RENiBEl [8], a consortium of university laboratories funded by the European Commission in a Fifth Framework Research Training Network. The RENiBEl RTN published more than fifty papers during the lifetime of the project and its members made more than 150 conference presentations. It is not the purpose of this review to provide exhaustive details of everything that was done by each of the collaborators and trainees during the project. In this summary of the highlights, we first sketch the theoretical underpinning that is used to describe RE ions, their energy levels and optical interactions with a semiconductor host; in the succeeding section, we describe conventional methods used to prepare III-N:RE epitaxial samples; we review definitive characterisation studies of RE in (mainly) implanted samples, discuss recent theoretical modeling of RE lattice location in nitrides and briefly consider the excitation mechanisms; we finally discuss the 'defect problem': how to compare the results of the lattice location studies with purely spectroscopic evidences of site multiplicity and thereby attempt to identify the strongly emitting sites.

An abbreviated early version of this review was presented at the Fall Meeting of the Materials Research Society in December 2004 and was subsequently published in the Proceedings of that Symposium [9].

\section{Theoretical background}

Theoretical treatments of $\mathrm{RE}$ ions in nitrides are carried out for a variety of purposes. In this section we review the basic chemistry and physics that serves as a background to these efforts, with emphasis on the labeling of states that are involved in optical transitions and the calculation of the relevant energy levels and transition strengths.

\section{$2.14 \mathrm{f}$ term symbols and spectra}

For "mildly" relativistic atoms such as the lanthanide series, the atomic states can be labeled with term symbols appropriate to Russell-Saunders coupling, ${ }^{2 S+1} L_{J}$, with the term multiplicity $2 S+1$ and the total angular momentum of $J$ (in units of $\hbar$ ).

Each term symbol labels a degenerate group or multiplet of $2 J+1$ states (which may be split by an external field). In the lanthanides, $L$ and $S$ are reasonably good quantum numbers, and permissible values of $J$ fall between $|L+S|$ and $|L-S|$, with Hund's rules providing a reasonable ordering of the energy levels in most cases. Since the $4 f$ shell is localised and distinct from the states of the surrounding crystal, it is customary to label the states by considering only multiplets of the $4 f$ manifold. Van Vleck [10] pointed out that $4 f \rightarrow 4 f$ transitions are forbidden in the free ion, because RE luminescence comes from dipole transitions and the operator for these is of odd parity. Judd [11] and Ofelt [12] independently proposed that the crystal field surrounding a RE ion in a host could produce mixed states that contain contributions from two 
(or more) configurations of electrons (typically $4 f^{n}$ mixing with $4 f^{n-1} 5 d$ ), if (and only if) the ion's environment lacks inversion symmetry. (The Ga substitutional site in GaN has $\mathrm{C}_{3 v}$ symmetry, which is non-centrosymmetric.) This consideration formally invalidates the practice of labeling the $4 f$ states with the free atom term symbols.

In a configuration-interaction picture of the $4 f$ states, combinations of all determinants for the $n$ electrons in $N$ spin orbitals give the resulting multiplets. (Frequently, only the $4 f$ spin orbitals are used to set up these multiplets, leaving out the $5 d$ contribution.) The resulting states are further split by the surrounding crystal- and ligand-field interactions (Stark splitting) which depend on the local site symmetry of the RE surroundings. The resulting states can be labeled more properly, as suggested by Racah, as irreducible representations $\left(\Gamma_{i}\right)$ or equivalently by using the Mulliken point group symbols [13]. More arcane symbolisms exist; however when the actual site symmetry of the RE ions is unknown, or when one wishes to compare different sites in a host, the inaccurate and familiar term symbols give labels that are at least a first approximation to the states involved.

Models of the $4 f$ states and their transitions have been considered by techniques ranging from the phenomenological to ab initio. Extensive measurements in the 1950s and 60 s of the $4 f^{n}$ configurations of the lanthanides in lanthanum fluorides and other hosts, much of them done by Dieke [14], gave a consistent picture of energy levels for trivalent lanthanides. The resulting set of levels (sketched as the "Dieke diagram") is useful since the energies of RE multiplets vary only fractionally between different hosts.

\subsection{Modelling of luminescence from rare earths in solids}

At the most empirical level, a form of Judd-Ofelt theory can be used to express dipole allowed transition intensities for a given RE ion in a host (for example, in solution, or in a vitreous or crystalline environment). In the simplest form of the theory, the integrated intensities of a transition between two multiplets, $a$ and $b$, for a given RE environment, are proportional to $\sum_{\lambda=2,4,6} \Omega^{\lambda}\left\langle a \| U^{\lambda}|| b\right\rangle^{2}$, where the three adjustable parameters, $\Omega_{\lambda}$, quantify Coulomb interactions between $f$-electrons, and the double-barred matrix elements belong to the reduced unit tensor operator [15], which depends in turn on the crystal and ligand fields. Additionally, the spin-orbit interaction of the $4 f$ electrons is also normally fitted. Given a sufficiently large set of identified transitions between different multiplets, the $\Omega_{\lambda}$ and spin orbit parameters, and the surrounding crystal field can be determined [16] (the number of observed lines needed to give reliable fitting leads to an overdetermined fit). Some care is necessary in both the fitting and also the use of predicted intensities [17]. In particular the transitions used in fitting must all belong to the same site, which becomes problematic in the case of the doped III-nitrides as discussed below. Typically the crystal field is expanded in a linear combination of products of angular and radial parts, the choice of terms being set by the local site symmetry of the RE ion; the number of terms naturally increases with reduction in symmetry. The level of sophistication for the crystal field calculation can vary from a point charge [18] model to the use of density functional theory (DFT) [19].

\subsection{Modelling of sites}

Our main concern, whether as experimentalists or theorists, is to identify particular sites that are important (i.e. bright) luminescent centres. As discussed above, the correct modeling of $4 f$ states of $\mathrm{RE}$ atoms in solids presents some challenges, in part deriving from the multiconfigurational nature of the states involved. Large-scale configuration-interaction based calculations become prohibitively expensive if large numbers of host atoms are included, but methods based on a mean-field DFT approach give qualitatively incorrect descriptions of strongly localized states, allowing far too much hybridization with their surroundings [20].

Within the RENiBEl network, two types of DFT model, with different methods for treating the $4 f$ shell, have been applied in order, first of all, to establish the relative stability of different site structures. Both types of approach developed from treatments of RE ions in silicon by these and other groups, but it is only recently that RE impurities in compound semiconductors have been investigated by such methods.

The Jones group at the University of Exeter used the AIMPRO code [21] to calculate the energetics and electrical properties of Er, Eu and Tm in a variety of semiconductor hosts. The problematic RE $4 f$ shell was treated as core-states of a pseudopotential that was generated for appropriate trivalent configurations of the ions. More details can be found in Filhol et al. [22]. The Frauenheim group in Paderborn used "LDA+U" techniques [23] that include a self-energy correction for the $4 \mathrm{f}$ states which is derived from the Hubbard model either in an extended form of the spin-polarized density-functional based tight-binding (DFTB) method [24] or using the all-electron WIEN2k code [25]. Focusing on $\operatorname{Er}_{\mathrm{Ga}}$, the Paderborn group found that in the local-density or regular DFTB approximations a degenerate group of localized $f$-related states appears near the middle of the gap. Applying the LDA+U potential pushes the occupied states downwards in energy into the valence band while the empty states are pushed upwards into the conduction band (CB), leaving the original band gap completely empty [26].

An alternative DFT-based method that has recently been applied to isolated $\mathrm{RE}_{\mathrm{Ga}}$ includes a correction for the self-interaction error present in the usual functionals [27]. Svane et al. broadly agree with our conclusions that $\mathrm{RE}^{3+}$ is the dominant charge state, with an acceptor level lying above the conduction-band minimum. They predict that $\mathrm{Eu}_{\mathrm{Ga}}$ possesses an acceptor level that is above the calculated CB minimum, but lies within the experimental gap. 


\section{Sample preparation}

Within the RENIBEl network, samples were prepared in one of two ways, either by in-situ doping during MBE growth or by ion implantation into previously grown material. MOVPE at the University of Montpellier in France was first used to grow GaN templates of high quality. Insitu doping of nitride samples during MBE growth was carried out with MOVPE templates employed as pseudosubstrates. MBE layers can be both considerably thicker and more heavily doped than implants, which may prove useful for certain purposes. Implantation of high-quality epitaxial layers by $\sim 100 \mathrm{keV}$ ions, usually aligned with the major crystal direction coincident with the surface normal, results in the production of a thin layer $(\sim 100 \mathrm{~nm})$ of doped material. The crystal lattice is grossly disrupted by the implantation damage and annealing is usually required to obtain luminescence from the implant. Implantation/annealing experiments were carried out either at ITN, Lisbon in Portugal or at IKS, University of Leuven, Belgium. Additionally, a few implanted GaN:Eu layers were obtained from the University of Toyohashi in Japan (Yoshida-Wakahara laboratory) and a set of MBE-grown GaN:Er layers from the University of Cincinatti (Steckl nanolab.)

\subsection{In-situ doping}

Samples with direct in-situ doping of RE were produced by the use of Gas Source Molecular Beam Epitaxy (GSMBE) with ultra-pure solid RE metallic sources. Substrates were either $p$-type Si (111) wafers with a thin $(\sim 10 \mathrm{~nm}) \mathrm{GaN}$ or AlN buffer or MOVPE-grown GaN templates on sapphire. Growth temperatures ranged from $600{ }^{\circ} \mathrm{C}$ to $900{ }^{\circ} \mathrm{C}$. By adding the impurity directly during GaN growth, it was possible to avoid the lattice damage induced by ion implantation and to produce samples with an extremely good crystalline quality and a very wide range of doping concentrations. However, there is some experimental indication that structurally good samples show rather poor luminescence [28].

A wide range of growth conditions was investigated and samples with degraded crystalline quality were deliberately grown. We demonstrated that the best photoluminescence results were not correlated with the crystalline quality of the samples and reached the conclusion that some unidentified defects were involved in the energy transfer from the host matrix $(\mathrm{GaN})$ to the rare earth ions in MBE-grown samples [29].

\subsection{Implantation of samples}

High quality epitaxial layers of $\mathrm{GaN}$ were grown by MOVPE on sapphire substrates for ion implantation. This enabled the consortium to produce RE-implanted GaN layers, and compare them with epilayers doped in-situ. High temperature annealing of the implanted samples was usually necessary to observe any RE luminescence. The intensity of the RE bands at first grows with increasing annealing temperature and then falls off as irrecoverable sample degradation occurs. During the course of our annealing studies, it became clear that it would be of use to produce GaN templates protected with a thin "nanocap" layer of AlN. Using these samples, it was possible to implant rare earth ions into GaN, through the AlN cap, which, thanks to its high thermal stability, allowed us to perform extreme annealing (at temperatures in excess of the template growth temperature) after implantation $[30,31]$.

Material implanted at room temperature does not often show any RE-related luminescence, which typically appears only after an extended high temperature anneal. Two processes that may enhance RE emission are the removal of efficient non-radiative recombination centres, and the formation of specifically efficient RE-containing lumophores. Since the rare-earth luminescent lifetime is relatively long, few non-radiative routes for de-excitation must be present in the material to allow production of light at the targeted transition energies, and competing radiative processes must also be suppressed. Additionally, if a specific lattice location (or locations) is required for the RE ions to produce efficient luminescence, annealing may allow diffusion of the ions to occur. However, changes in site occupation with annealing are more likely to be caused by interaction with defects or ion clustering [32].

A number of stable or radioactive $\mathrm{RE}$ ions were implanted into an extended range of group III-nitride substrates, i.e. GaN, AlN, InGaN and AlInN. Fluences between $10^{12}$ atoms $/ \mathrm{cm}^{2}$ and $10^{16}$ atoms $/ \mathrm{cm}^{2}$ were used, with energies between 60 and $380 \mathrm{keV}$, at sample temperatures ranging from 20 to $800{ }^{\circ} \mathrm{C}$, and with different implantation geometries - that is, to achieve random or channeled implantation. The aim of this approach was to understand (i) the defect build-up, amorphisation process and damage recovery, and (ii) the lattice site(s) of the implanted species, depending on the specific implantation conditions.

During annealing several additional processes can impact on the luminescence efficiency. Amorphisation of the lattice can occur under higher implantation fluxes, but was not particularly evident in our studies [33,34]. Annealing of an amorphous layer may lead to its evaporation or to the formation of a polycrystalline layer. In our studies, surface amorphisation was effectively suppressed either by use of an AlN cap or by implanting at higher temperatures.

An investigation has been made of the effects of varying the substrate temperature and ion fluence during ionimplantation. CL measurements show a linear increase in luminescence intensity with increasing substrate temperature, up to $450{ }^{\circ} \mathrm{C}$. On the other hand, the CL intensity increases linearly with the ion fluence for low fluence and saturates for higher fluences (where the defect accumulation becomes significant). Both tendencies show a remarkable resemblance with the (absolute) number of substitutional erbium ions as a function of the substrate temperature and the ion fluence, respectively. This resemblance suggests a correlation between the origin of the 
luminescence and the doping density - although lattice damage is claimed to play a crucial role in the luminescence process of the implanted rare-earth ions.

The defect concentration is found to decrease with increasing substrate temperature during implantation, due to an improved dynamic annealing effect in which the mobility of the defects is enhanced, thus resulting in more defect annihilation. Implantation into a substrate at above $65{ }^{\circ} \mathrm{C}$ gives better crystalline quality than a room temperature implant followed by a $950{ }^{\circ} \mathrm{C}$ anneal. This further suggests that the combination of an elevated temperature and excitation from the implantation (whether through electronic excitation or additional heating) have a synergistic effect on recovery from implantation.

Three damage accumulation stages are found with increasing implantation fluence: a slow initial build-up, followed by a steep increase, and finally saturation of the damage concentration [35]. In the case of channeled implantation, the second and third regions are shifted to significantly higher fluences. Moreover, in the first and second region, the substitutional fraction of the implanted species is found to decrease linearly with increasing defect density, while the maximum induced strain increases with defect density. Both relationships exhibit the same linearity, independent of the ion fluence, the implantation energy, the substrate temperature during the implantation and implantation geometry. Hence, they are an intrinsic property of the GaN lattice.

\section{Sample characterisation}

In this section we describe first those large-facility characterisation techniques used to establish the lattice location of impurity ions in solids. We then illustrate the use of spectroscopy to reveal the existence of multiple optical sites, which suggests a distribution of RE lumophores with distinct optical spectra, which we assume to be representative of populations of ions in (a small number) of different environments.

\subsection{Electron emission channeling (EC)}

A convenient way to determine the lattice site of impurities is by making use of the channeling effect. However, conventional channeling techniques based on the use of probe ion beams, like Rutherford backscattering spectrometry/channeling (RBS/C) or particle-induced X-ray emission (PIXE), require the presence of a large concentration of impurities in the material. A large impurity concentration has the disadvantage that it might also introduce unwanted effects, such as clustering of the impurities, or crystal damage if implantation is used to introduce the foreign elements. One channeling technique that can be used to investigate low concentrations of isolated impurities is electron emission channeling (EC).

Lattice location studies by electron emission channeling seek to determine the sites occupied by implanted radioactive ions, production of which requires access to a

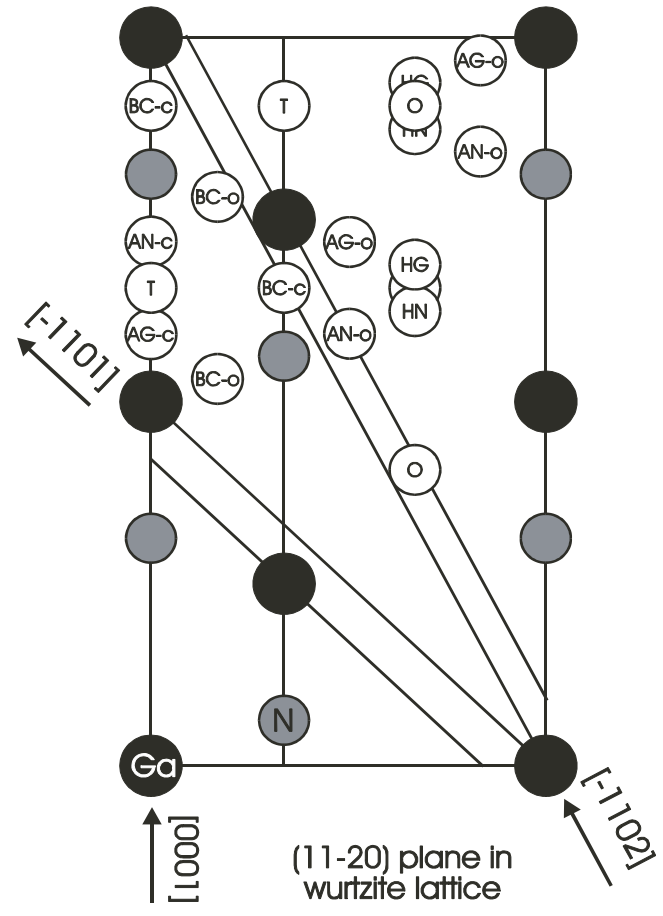

Fig. 2. High-symmetry sites of the wurtzite lattice. Ga and N atoms in the perfect GaN crystal are represented by black and grey filled circles, while the various interstitial sites, in white, are labelled conventionally: $\mathrm{O}$ for octahedral and so on.

gigavolt proton source. EC measures the angular distribution with respect to major crystal axes of $\beta$ - or conversion electrons (from daughter nuclei) that are emitted by the implanted ions during their radioactive decay. The experimental set-up used in our experiments at CERN is unique in its deployment of two-dimensional electron detectors to measure the emission patterns [36]. A radiative heater is incorporated in the set-up to allow in-situ annealing studies.

The physical basis of EC is the guiding of the ejected electrons by rows of positive nuclei along (the) main crystalline directions; the electron yield increases if the emitting atom is "in line". Simulation compares patterns calculated for emission by atoms in certain high-symmetry sites with the results of experiments. Some possible sites in the wurtzite lattice that should be considered in such simulations are indicated in Figure 2. A key observation is that the patterns produced by different sites are very easily distinguishable from one another in EC studies.

The majority (50-95\%) of Pr, Nd, Eu, Gd and Er ions, implanted into GaN at low fluences $\left(\sim 10^{13} \mathrm{~cm}^{-2}\right)$, occupies lattice sites that appear to be slightly displaced from the perfect substitutional $\mathrm{Ga}$ site, $\mathrm{RE}_{\mathrm{Ga}}$, with a (nearly) isotropic root mean square (rms) displacement of the order of $0.10-0.25 \AA$. The remainder of the implanted ions is assumed to reside on low-symmetry sites or lattice sites with more disordered surroundings. 
After high-temperature annealing, the substitutional fractions stay nearly constant, while the rms displacements decrease, indicating that the measured displacements are most likely related to the presence of a random distribution of implantation-induced defects in the vicinity of the impurity atoms. The fact that the displacements are observed for all investigated impurities, and that none of the impurities exhibit perfect incorporation for annealing temperatures below about $1200{ }^{\circ} \mathrm{C}$, indicates that the formation of impurity-defect complexes for substitutional impurities is a universal process in $\mathrm{GaN}$ and that the corresponding binding energies must be fairly large. This observation can explain both the site multiplicity in luminescence experiments on rare earths (see Sect. 4.3 below), and the requirement of very high annealing temperatures in order to activate implanted dopants in GaN despite the fact that the majority of them are already located on substitutional sites in the as-implanted state. The natural candidates for the enhancing defects are $\mathrm{N}$ vacancies in the nearest neighbor shell of the substitutional impurity, Ga vacancies in the second neighbor shell, or Ga interstitials between the first and third neighbor shells.

Additionally, we have studied the influence of external parameters, besides the annealing temperature, on the lattice site location of erbium in GaN. It was found that coimplanting erbium together with oxygen or carbon does not result in the occupation of fundamentally different lattice sites of Er [37]. In fact, the differences between the co-implanted and undoped GaN samples are within the experimental error bars, which means that any difference in luminescence caused by co-implantation of Er with $\mathrm{O}$ or $\mathrm{C}$ into $\mathrm{GaN}$ (either an increase or decrease; both have been reported) cannot be attributed to a change in the lattice site of Er, in possible contrast to the case of Er in silicon.

To summarise, in a large number of studies performed to date, there is no indication of $\mathrm{RE}$ on any highly symmetric lattice site other than the substitutional Ga site $[28,38]$; rms displacements of RE ions, an adjustable parameter in the simulation procedure, seem to be much larger than one would expect if only thermal vibrations were present; this additional displacement is most likely to be attributable to the surrounding crystal disorder. However, in all cases the rms displacements decrease after annealing, indicating better on-site incorporation of the RE, or the removal of perturbing defects, which is more likely. At the same time there is an increase in the efficiency of RE luminescence, which indicates at least a correlation between crystal perfection and luminescence output for RE-implanted samples. Additional experiments [39] have investigated high-fluence implantation and channeled implantation of Er into GaN, and confirm the important role of implantation-induced defects.

\subsection{X-ray absorption fine structure (XAFS)}

XAFS determines the lattice sites occupied by native or impurity ions in crystalline solids by exploring local structure interactions [40]. What is measured in this technique

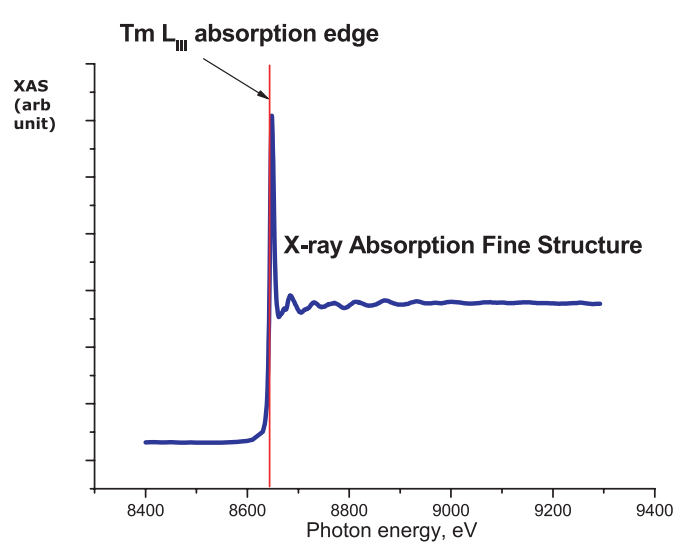

Fig. 3. The X-ray absorption spectrum (XAS) of a Tm-doped GaN sample shows the characteristic $L_{\mathrm{III}}$ edge of $\mathrm{Tm}$, above which appear the fine-structure oscillations of XAFS (see text).

is the absorption spectrum in the vicinity of characteristic X-ray ionisation 'edges' due to particular elements, for example the $L_{\mathrm{III}}$ edge of Tm at $8.64 \mathrm{keV}$. The investigation of such absorption spectra requires the use of a bright, continuously tunable X-ray source, such as an electron synchrotron. Our experiments used the UK Synchrotron Radiation Source at Daresbury Laboratory. The X-ray absorption spectrum (XAS) of a target atom depends upon its local environment. Interference of the emitted and reflected photoelectrons introduces a characteristic series of oscillations (Fine Structure) above the edge. An example of such a spectrum is shown in Figure 3.

A Fourier Transform of the XAFS oscillations yields the RDF (radial distribution function) of the ions that neighbour the target atom. Weighted RDFs are shown in Figure 4 for two GaN:Tm samples with rather different doping concentrations. Whereas impurity aggregation effects are expected and observed in heavily doped GaN:RE [41], XAFS clearly reveals aggregation in Tm-doped GaN at rather low levels of doping. Tm atoms are found only in substitutional Ga sites for low concentration samples $(<0.5 \mathrm{at} \%)$. When the concentration is increased to $1-$ $2 \mathrm{at} \%$, Tm is found again on the Ga substitutional site, surrounded by 4 nitrogen atoms, but the presence of a substantial number of Tm ions in the second coordination sphere indicates the onset of dopant clustering in these films. In the more strongly doped sample, the split nitrogen shell indicates the presence of actual phase decomposition: a sizeable proportion of the Tm ions find themselves in a TmN environment in this sample [42]. The formation of pure TmN clusters was found in an in-situ doped sample with a dopant concentration as low as $3.4 \%$.

It should be noted that XAFS, in contrast to EC, is not particularly sensitive to random displacements of a target atom from the centre of its lattice site: such deviations are averaged out in the summing of contributions to the X-ray absorption signal from different atoms, and lead to a broadening of the RDF, which is parametrised in the fitting procedure as a Debye-Waller factor. Any comparison of lattice disorder using EC and XAFS would first require 

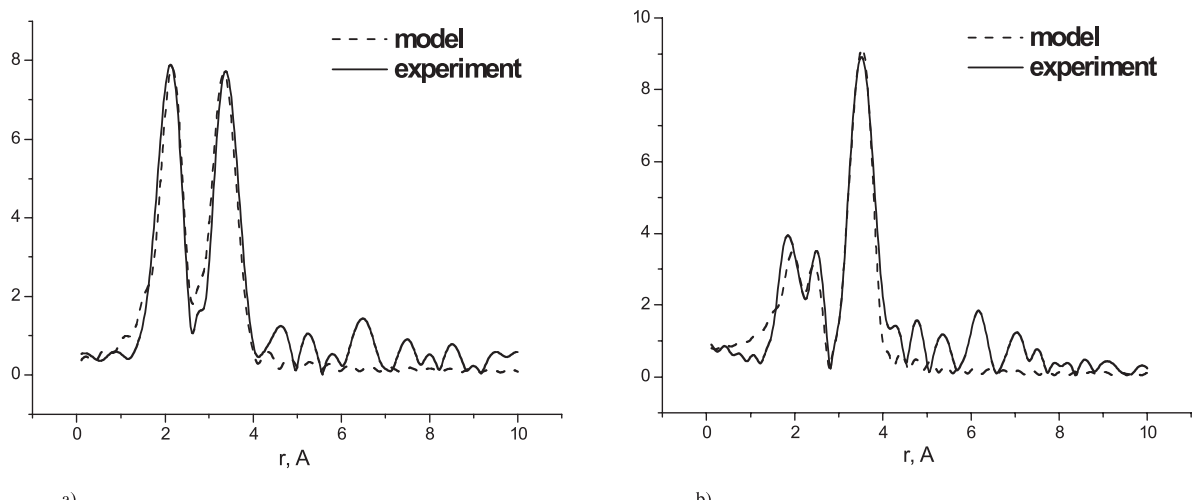

Fig. 4. A Fourier transform of the XAFS oscillations yields the radial distribution function for atoms surrounding the target, in this case Tm in GaN at concentrations of (a) $0.5 \%$ and (b) 3.4\%. The splitting of the first shell for the more concentrated sample indicates the formation of TmN clusters within the sample.

a disentangling of the dynamic thermal contributions from those due to static atomic displacements.

To summarise the XAFS results obtained to date, all $\mathrm{RE}$ ions studied have been found to reside on Ga substitutional sites [43]. The lattice location was found to be independent of the doping method used to produce the samples. In contrast to the case of In atoms incorporated in InGaN, RE in GaN were found to have a strong tendency to agglomerate: when the RE concentration increases above a fairly modest threshold, REGaN clusters with locally high RE content are observed; the lowest concentrations at which RE clustering was observed are $0.17 \%$ and $1.2 \%$ for $\mathrm{Er}$ and Tm, respectively. At a still higher level of doping, pure REN phases are observed. The stoichiometry of the growth conditions decisively influences the phase segregation: in the case of the Er-doped samples, a lack of $\mathrm{Ga}$ under N-rich growth conditions leads to an enhanced incorporation of Er into Ga sites at the expense of the formation of Er-rich clusters and the ErN phase [33].

\subsection{Optical studies (PL, PLE, CL, time-resolved)}

It is not surprising that most previously reported work on epitaxial RE-doped semiconductors has focused on their optical spectroscopy. However, the simplest and most direct technique of transmission (absorption) spectroscopy is seldom applied to these samples: the doped layers are usually too thin, the samples too dilute and the transition strengths too small for experimental success to be guaranteed.

The 'strength' of a particular spectral transition is conveniently normalised to that of the same number density of classical electromagnetic oscillators. The 'oscillator strength' or ' $f$-number' of a classical oscillator is 1 and the decay time of excitation for such an oscillator (a result of radiation damping) is about $1 \mathrm{~ns}$. RE ions in nitrides typically have radiative lifetimes, measured at low temperatures, of about $100 \mu \mathrm{s}$ : this figure equates to an oscillator strength of $10^{-5}$. The total optical effect of $N$ sites (per unit volume) is then given by Smakula's product, $N f$.
An alternative to absorption spectroscopy is the detection of luminescence, which may be excited in one of a variety of ways; PL and CL are the most commonly used techniques to obtain spectroscopic signatures of particular RE ions; while PL is spectrally selective, depending on the photon energy of excitation, CL tends to 'excite everything' that the high energy electron beam encounters; fortunately, the penetration of an electron beam can be restricted by limiting its energy. Photoluminescence excitation (PLE) spectroscopy monitors the intensity of a particular PL emission feature, a line or a band, for example, as the exciting light is swept through a range of higher photon energies; it produces spectra that are sometimes comparable to those that would be obtained by the transmission technique (quasi-absorption spectra) but with much higher sensitivity [44]. Finally, both PL and CL can be performed in time-resolved (TR) mode: when the sample excitation is removed, the luminescence signal decreases towards zero as the excited state population returns to its (small) thermal equilibrium value. The decay rate of a particular energy level has a radiative component, as described above, and a non-radiative part, which is usually strongly dependent on temperature.

In almost all cases reported to date, luminescence spectroscopy of III-N:RE samples offers clear evidence for the co-existence of a number of different sites with distinctive excitation and emission signatures. Dierolf et al. [45] have provided a very clear recent example of site-selective spectroscopy of MBE-doped GaN:Er; wavelength-stepped laser excitation and recording of luminescence spectra by a CCD-spectrograph, produces Combined ExcitationEmission Spectra (CEES). Different luminescent sites may be readily identified in these spectra by the coincidence of excitation and emission lines in particular groupings; Dierolf et al. find two 'majority' and up to four 'minority' groupings in CEES spectra of GaN:Er, in broad agreement with previous results $[46,47]$. These observations mainly concern internal transitions of the RE ions, below the host band-gap in terms of photon energy, with some sites appearing to require 'feeding' by others (intersite energy transfer). At the same time, the sites are observed to interact differently with the host (which is 

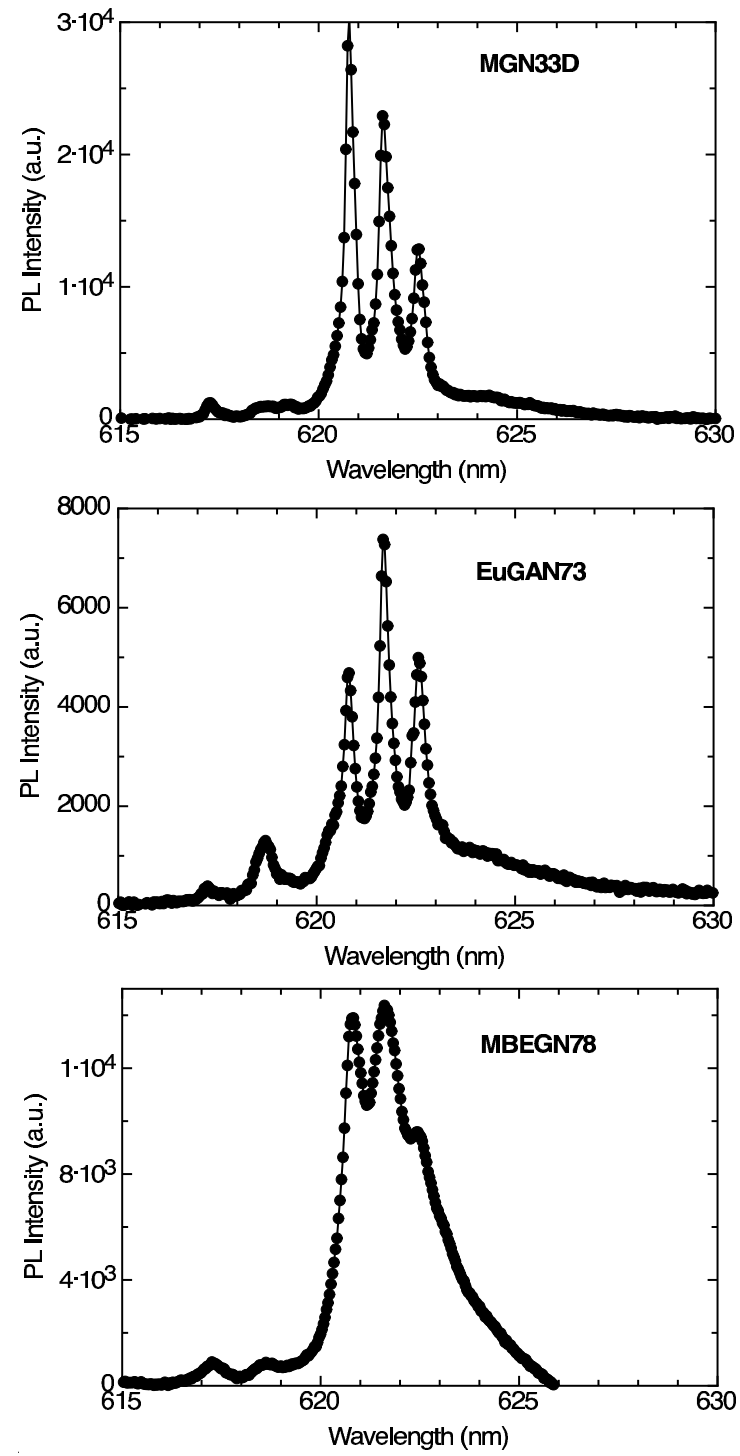

Fig. 5. PL spectra of various GaN:Eu samples show that the splitting pattern of the $622 \mathrm{~nm}$ spectral multiplet is sampledependent. This gives a clear visual indication of the existence of site multiplicity in Eu-doped GaN.

itself excited through band-to-band transitions that create electron-hole pairs).

As an example of the use of PL/PLE to reveal excitation mechanism, we summarise here some recent spectroscopic work done on $\mathrm{GaN}: \mathrm{Eu}$, which emits predominately red light, near $622 \mathrm{~nm}$, in a transition identified as ${ }^{5} D_{0}-{ }^{7} F_{2}$. (The transitions ${ }^{5} D_{0}-{ }^{7} F_{1}$ and ${ }^{5} D_{0}-{ }^{7} F_{3}$ produce similar but weaker lines near $600 \mathrm{~nm}$ and $633 \mathrm{~nm}$, respectively, according to the general rule that $(J=0) \rightarrow$ $\left(J^{\prime}=\right.$ odd $)$ transitions are only weakly allowed [48].) Highresolution PL spectroscopy at low temperature previously showed that the $622 \mathrm{~nm}$ line is in fact spectrally complex [49]: the line has at least 3 components. A crystal field splitting may lead to a line splitting, of course, without different sites being involved, but not in this case [50]. The first evidence for site multiplicity in GaN:Eu emerges

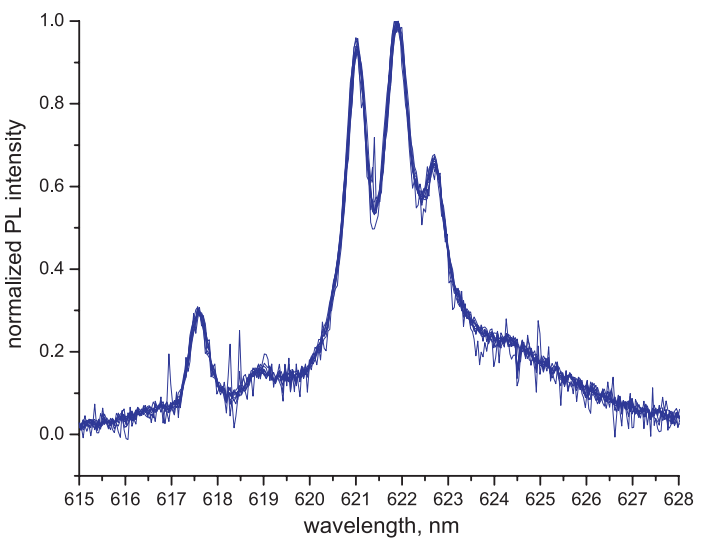

Fig. 6. Presenting a contrast to Figure 5, confocal micro PL, taken at 10 different locations on a single sample of GaN:Eu at $140 \mathrm{~K}$, shows very good uniformity of the splitting pattern of the $622 \mathrm{~nm}$ luminescence line.

from a comparison of PL spectra of a number of different samples taken under the same conditions of excitation: $5 \mathrm{~mW}, 325 \mathrm{~nm}, 15 \mathrm{~K}$; a selection of such spectra is shown in Figure 5: samples prepared differently show measurably different spectral profiles. On the other hand, the consistency of the luminescence profile, obtained by using a confocal microscope at 10 different positions on the surface of a given sample at $140 \mathrm{~K}$, shows the very good uniformity of ion distribution achievable by consistent preparation (Fig. 6). A comparison of PL spectra at high and low temperatures next reveals very different temperature dependences for the lines of the $622 \mathrm{~nm}$ triplet: the line at highest energy, dominant in most samples at low temperature, vanishes near $250 \mathrm{~K}$, while the other two lines of the triplet reverse the ordering of their relative intensities. At the same time, a new line (also showing some internal structure) increases in intensity at $617 \mathrm{~nm}$; between $13 \mathrm{~K}$ and $140 \mathrm{~K}$ it appears to "borrow" intensity from the main triplet before it also quenches at higher temperature.

Whereas the excitation by laser light used in PL addresses the whole of the doped region in a typical sample, the penetration into a solid by an electron beam in CL depends strongly on the beam energy. For example, a $3 \mathrm{keV}$ beam excites material to a depth of about $80 \mathrm{~nm}$ in $\mathrm{GaN}$ while an $8 \mathrm{keV}$ beam reaches $330 \mathrm{~nm}$. Depth resolving CL spectroscopy at $15 \mathrm{~K}$ reveals some telling differences in the response of different components of the $622 \mathrm{~nm}$ multiplet, which are shown in Figure 7 . The line near $621 \mathrm{~nm}$ shifts to higher energy with increasing electron beam energy; the shift is more noticeable in more deeply implanted samples (at 350 vs. $75 \mathrm{keV}$ ). This depth dependence of the transition energy may point to a correlation of strain with the dopant or defect density. The relative intensities of the various lines also show a clear variation with depth and there is some indication of an unresolved background contribution to the luminescence. The best fit to the spectral profiles for all samples was achieved using four Lorentzians with peaks at 620.5, 621.4, 622.3 and $624 \mathrm{~nm}$, the last being considerably broader than the 


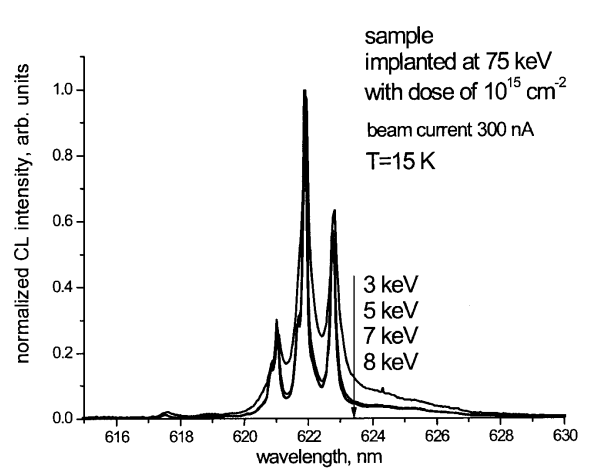

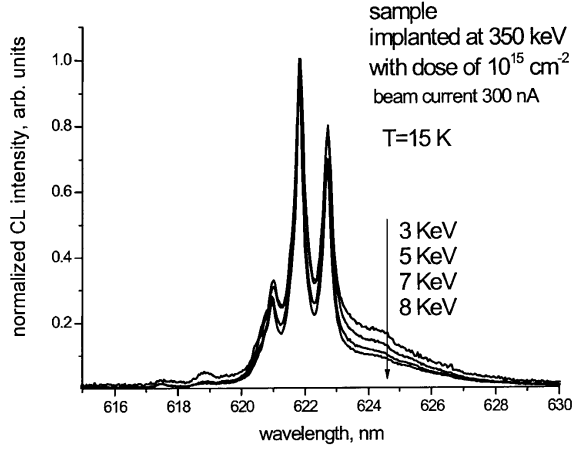

Fig. 7. CL profiling of (a) shallow and (b) deep implants reveal subtle depth dependences of the $622 \mathrm{~nm}$ emission from GaN:Eu (see text).

others. These components are the same as those observed in low-temperature PL.

Finally, the dependence of the PL line pattern on the excitation wavelength provides clear evidence for site multiplicity in GaN:Eu. In Figure 8a the line pattern changes decisively when the excitation wavelength crosses the lowtemperature band-gap of GaN near $355 \mathrm{~nm}$; for below-gap excitation, an additional major component of the luminescence multiplet reveals itself as a shoulder at $621.6 \mathrm{~nm}$; excitation below the band-gap also appears to sharpen all of the lines. In the excitation spectra of Figure 8b we identify a band edge feature, common to all lines of the triplet, and a below-gap plateau. Additional PL lines appear in the plateau region (Fig. 8c). From the evidence we identify the existence of a pair of doublets, only one of which (at $621.6,622.3 \mathrm{~nm}$ ) is excited below the gap; these doublets clearly belong to two different sites. The broad 624 $\mathrm{nm}$ line belongs to another site, while the $617 \mathrm{~nm}$ line, observed only at intermediate temperatures, remains unexplained. The behaviour of the small lines below $620 \mathrm{~nm}$ is even more complicated. Hence, the differences observed in the additional fine structure in the PL and CL spectra of GaN: $\mathrm{Eu}$ indicates the existence of at least four luminescent sites, $[51,52]$.

To summarise, PL and PLE studies indicate that above band gap excitation can simultaneously excite different types of defect in GaN:RE samples, leading to the emission of RE ions with characteristic PL signatures. Some sites can be excited selectively by carefully choosing the excitation wavelength below band-gap. In Eu-implanted GaN samples and in-situ Eu-doped MBE samples we observe two dominant types of luminescent centres [30,53]. We infer that the same or very similar Eu centres are present in both implanted and MBE samples.

\subsection{Supplementary characterisation techniques}

The surface morphology of samples was routinely assessed using scanning electron microscopy (SEM) at moderate resolutions, complemented in some cases by High Resolution Transmission Electron Microscopy (HRTEM) [54].
Raman spectroscopy was found useful in monitoring implantation damage and its recovery.

The doping concentrations of $\mathrm{RE}$ ions incorporated in GaN samples by in-situ doping or ion implantation has been accurately measured using Wavelength Dispersive X-ray (WDX) and Rutherford Back Scattering (RBS) spectroscopies; in both cases, RE contents below 0.1 at\% can be quantified accurately. RBS and WDX are found to be generally in good agreement as evidenced by measurement of the Eu content in a layer implanted with a nominal dose of $10^{14} \mathrm{~cm}^{-2}$, indicating 0.05 at\% within a layer only $\sim 40 \mathrm{~nm}$ thick: in the case of WDX, measurements as a function of electron beam voltage allow the thickness of RE-containing material to be estimated using a layer simulation software. More accurate depth profiling is possible with RBS, as has been demonstrated for GaN:Tm.

MBE-grown Tm-doped GaN samples have also been investigated with Atomic Force Microscopy (AFM). A strong influence of the growth temperature on the crystal quality and RE luminescence properties of these samples was found by an examination of surface features revealed by WDX and CL mappings [55].

Deep Level Transient Spectroscopy (DLTS) of implanted and annealed GaN samples show a level at $E_{C}-$ $0.2 \mathrm{eV}$, independent of the implanted species. Other levels were observed in the band-gap at $0.19,0.22,0.26$ and $0.65 \mathrm{eV}$ below the conduction band. The last two levels are found essentially in all of the investigated samples, suggesting that they are host-related (i.e. GaN growth defects). The first two levels appear exclusively in the random implanted and channeled implanted samples, respectively, which suggests a relation with the implantation geometry [56].

\section{RE doping of other III-nitrides}

The utility of the Dieke diagram in identifying transitions of particular ions in a variety of hosts points to the relative insensitivity of the actual transition energies to the crystalline environment. The much-studied IR band of $\mathrm{Er}^{3+}$ is 
found at, or near, $1.540 \mu \mathrm{m}$ in $\mathrm{GaN}, \mathrm{ZnO}, \mathrm{Si}$ and in every other host: the total reported variation in peak position that can be obtained by a change of host is rather less than $10 \mathrm{~nm}$ or $1 \%$.

Even when somewhat larger variations in transition energy are found experimentally (the dominant ${ }^{5} D_{0}-{ }^{7} F_{2}$ transition of $\mathrm{Eu}^{3+}$ can be found in the range from $610 \mathrm{~nm}$ to $625 \mathrm{~nm}$ ) it is by no means a straightforward matter to relate the relevant spectral fitting parameters to the chemistry and physics of the host material; in a wide-ranging investigation, Binnemans and Görller-Walrand found [57] that "a (certain) coordination polyhedron... results in weak crystal fields, regardless of the chemical nature of the ligands" (our italics). So, although the fitting of the line positions can be done precisely, the resulting fit parameters cannot be theoretically verified to a similar accuracy. One might expect on this basis that the comparison of RE spectra in GaN with those from other III-N materials (BN, AlN, InN and the alloys AlGaN, AlInN, for examples) might be a futile exercise. Happily, this is not the case; significant spectral changes are found when PL or CL of the same ion is examined in different III-nitride hosts. (The study of RE-implanted InGaN is hampered by its decomposition under high-temperature annealing; capping with a protective AlN layer may allow this investigation to proceed.)

AlN itself is even more resistant to radiation damage than GaN - fluences of $5 \times 10^{15} \mathrm{~cm}^{-2}$ introduce relatively little lattice damage; however, this damage is very stable and annealing at $1000{ }^{\circ} \mathrm{C}$ does not remove it $[58,59]$. Preliminary EC experiments [37] on the lattice site location of RE impurities in AlN have shown a situation that is quite similar to that of impurities in GaN; in both cases the majority of RE atoms is substitutional, although the impurities are somewhat displaced from the ideal lattice sites. Eu-implanted AlN shows fairly bright red luminescence. Probably due to the somewhat poor quality of available epitaxial layers, but we do not observe any fine structure in the ${ }^{5} D_{0}-{ }^{7} F_{2}$ transition of AlN: $\mathrm{Eu}^{3+}$. However, compared to similarly doped GaN the transition peak is shifted substantially to lower energy (from 622 to $624 \mathrm{~nm}$ ). Turning to AlGaN alloy, we note that substitution of RE on the Al and $\mathrm{Ga}$ sites may occur unequally. In any case alloy disorder will produce a wide range of crystal environments for dopants. Nakanishi et al. previously demonstrated [60] a strong influence of AlN content on the emission intensity of implanted AlGaN:Eu layers; for a similar series of alloys we found that the wavelength shift depends nonlinearly on the AlN content of the layers [61]. We have also performed the first investigations of RE-implanted AlInN, depth profiling Eu- and Er-implanted AlInN using time-of-flight secondary ion mass spectrometry (SIMS) and CL spectroscopy [62]. While all transitions broaden in the more disordered crystal fields of AlInN, those of $\mathrm{Eu}^{3+}$ shift to lower energy (compared to GaN) while those of $\mathrm{Er}^{3+}$, somewhat better resolved, shift to higher energies. More profound changes are associated with the spectra of Tm ions [63]; RTCL spectra of Tm-doped GaN, AlInN and AlGaN are compared in Figure 9. The blue luminescence
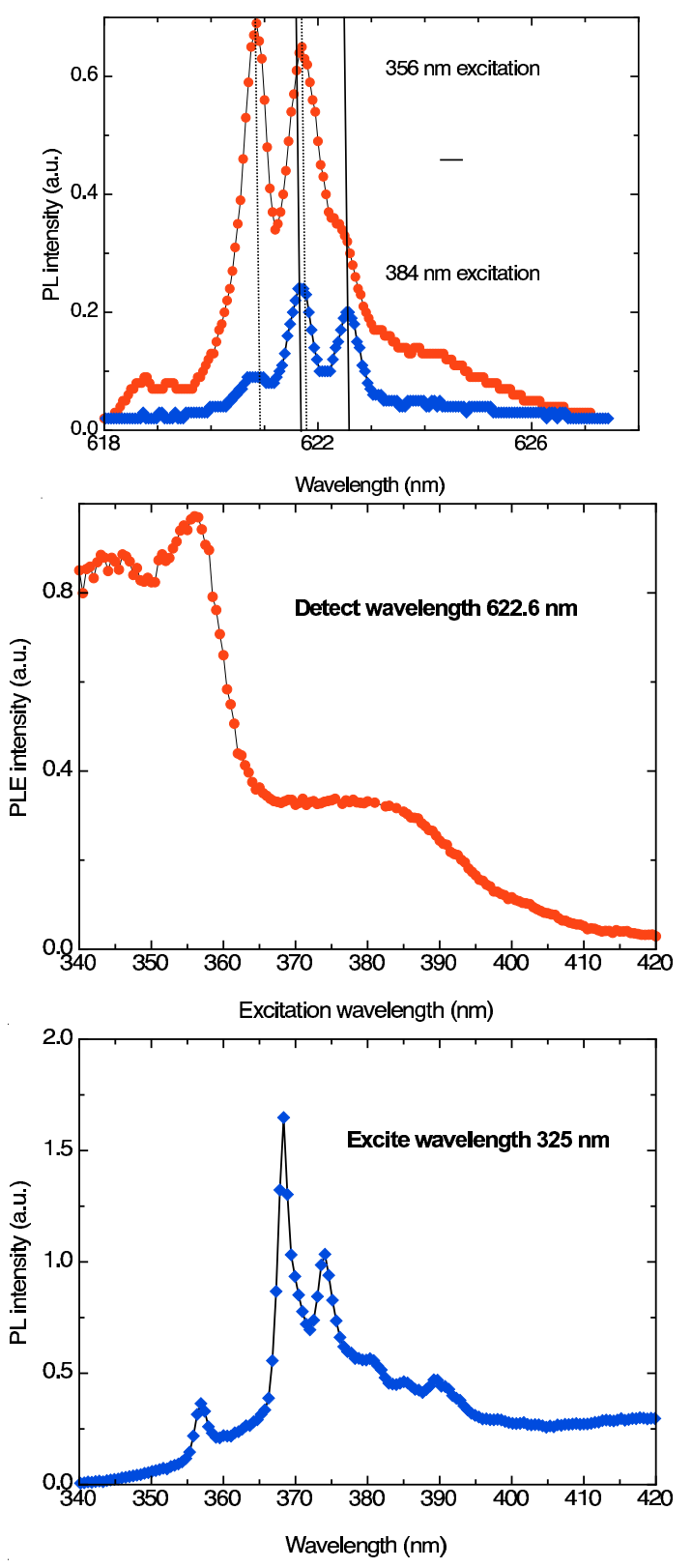

Fig. 8. Showing (a) the dependence of the $622 \mathrm{~nm}$ line profile on excitation wavelength, (b) the PLE spectrum of the $623.1 \mathrm{~nm}$ line, with a characteristic 'plateau' at $380 \mathrm{~nm}$ and (c) the PL in the plateau region of a sample excited above the GaN band-gap.

principally involves two bands of different origin that are favoured unequally in the different hosts; moreover, the integrated intensity of blue $\mathrm{Tm}^{3+}$ emission in AlInN is about 20 times greater than that in $\mathrm{AlGaN}$; It seems that the magnitude of the band-gap is not the sole determinant of the emission efficiency in this case.

\section{Discussion and conclusions}

It seems fair to comment that, despite many efforts, there has been to date no definitive assignment of any 


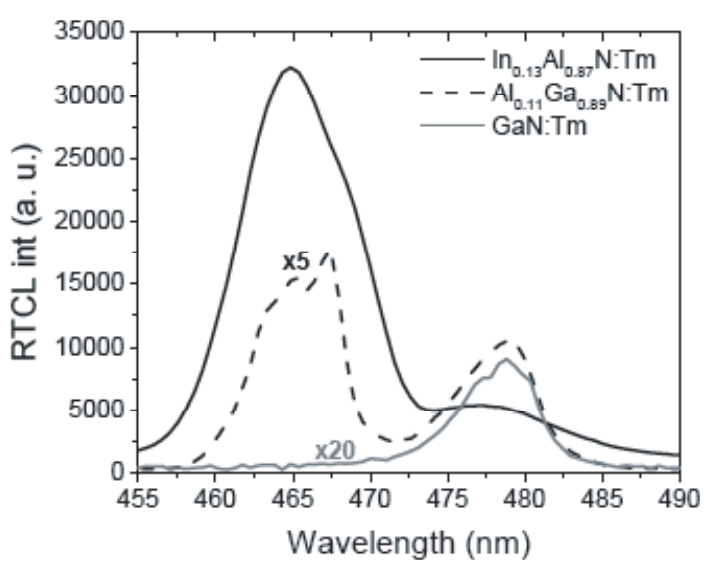

Fig. 9. III-N: Tm emission in the blue may involve two bands of different origin with peaks near $465 \mathrm{~nm}$ and $478 \mathrm{~nm}$, respectively. These bands are favoured differently in the GaN, AlGaN and AlInN hosts which are compared here.

luminescence band in RE-doped nitrides (or arguably in any semiconductor) to a particular site. Hence it seems clear that there is still a lot for both experimentalists and theorists to do.

The lattice location studies of RE-doped GaN performed by EC and XAFS agree that the majority of RE atoms occupy the Ga substitutional site. Theory concurs with this finding, adding that an interstitial RE atom is in fact less stable than interstitial Ga; RE interstitials will displace Ga substitutionals in equilibrium. Theoretical calculations conclude that isolated RE dopants in GaAs and GaN are electrically inactive (i.e. have no states in the gap) and thus require a close association with another defect to enable them to act as efficient carrier traps [64]. In contrast, $\mathrm{RE}$ ions in AlN are distinctive as they possess a deep donor level [65]. On the other hand, optical studies suggest that site multiplicity is the rule rather than the exception for RE atoms embedded in GaN. This contradiction raises questions about the lattice location of optically active centres (lumophores) in GaN and their emission efficiency, yet to be answered in detail both by theory and experiment.

Recent experimental support for the lack of electrical activity of isolated RE in GaN comes from work carried out both within the RENiBEl network and by others. GaN implanted with $\mathrm{Er}$ and $\mathrm{Pr}$, and subsequently annealed at $1000{ }^{\circ} \mathrm{C}$, possesses a free carrier concentration barely changed from that of the starting material and most significantly only about $1 \%$ of the RE concentration. This implies that the dominant RE site is electrically inactive. Moreover, the dominant defect detected by DLTS, as described above, near $E_{C}-0.2 \mathrm{eV}$, is present at concentrations of only $0.1 \%$ of that of the RE. The origin of these levels is at the moment obscure but according to theoretical models (see below) they may be related to $\mathrm{RE}_{\mathrm{Ga}}$ complexed with vacancies or oxygen. Deeper defects observed at around $E_{C}-0.6 \mathrm{eV}$ may also be related to RE. Further work needs to be carried out to try to correlate the populations of these defect levels with optical data.
An important side issue concerns the quenching mechanisms that affect RE luminescence in semiconductors. Several processes, verified for other semiconductor and inorganic hosts, may take place; among these one can find back-transfer of excitation from the RE ion to an exciton trap with a subsequent dissociation or recombination of the bound exciton which restores the ground state of the system; Auger processes where the excited RE ion transfers its energy to a free or trapped carrier are also thought to be of great significance [66]; finally, transfer of energy among RE ions can lead to a quenching of the emission [67]. All of these processes shorten the radiative lifetime. Experimentally, the measured lifetime becomes larger as the annealing temperature is increased in implanted GaN samples; for instance, annealing at $1300{ }^{\circ} \mathrm{C}$ compared to $1000^{\circ} \mathrm{C}$ reduces the impact of the quenching processes by an order of magnitude in GaN:Eu.

The overall efficiency of RE emission is quite low in nitrides; the optimum doping densities are found to be of order $1 \%$. Where site multiplicity is observed spectroscopically, as seems to be the case for Eu and Er in GaN, it seems quite unlikely that the pure substitutional defect $\mathrm{RE}_{\mathrm{Ga}}$ can be responsible for any of the observed luminescence spectra. If we focus attention on the Smakula product, $N f$, for each luminescent site, there appear to be at least 2 subtly different active centres in each example, with approximately equal $N f$ products: it is difficult to see how any one of these could be the pure substitutional site. Consider the example of the two strongly emitting sites in GaN:Eu. Sample treatment can favour population of one site or both, although no inter-conversion of sites during annealing has been reported. The two centres have approximately equal PL and CL intensities at low temperature, but show very different thermal quenching behaviour. While both can be excited via excitation transfer from the host (via above band-gap absorption), one shows an additional excitation pathway, perhaps involving the binding of an exciton at an energy just below the gap. If we choose one of these as the isolated site $\mathrm{Eu}_{\mathrm{Ga}}$, (and which one would you chose?), we must conclude that the other active centre, necessarily a lower symmetry site, is to be compensated for its lesser numbers by a luminescence efficiency that is greater in almost perfect proportion. The same argument will then apply down the chain of multiple sites. This is very hard to believe.

If the unperturbed RE ion does not emit light strongly, and there is no "magic site" which dominates the emission in each case, it seems likely that the observed multiple luminescence lines are due to centres with rather low populations and with more or less the same basic nanostructure. A prime candidate for such a family of sites is the set of $\mathrm{RE}_{\mathrm{Ga}^{-}}$(native defect) pairs: a large number of locations can accommodate $\mathrm{Ga}$ or $\mathrm{N}$ interstitials and vacancies, as shown in Figure 2. It is however difficult to associate particular emission lines with particular defect configurations. Filhol et al. [22] considered a number of energetically favourable complexes of native interstitials, vacancies and $\mathrm{O}_{\mathrm{N}}$ with $\mathrm{Eu}_{\mathrm{Ga}}, \mathrm{Er}_{\mathrm{Ga}}$ or $\mathrm{Tm}_{\mathrm{Ga}}$. $\mathrm{RE}_{\mathrm{Ga}}-\mathrm{V}_{\mathrm{N}}$ close pairs were calculated to have the largest binding 
energies (1.0, 0.8, and $0.7 \mathrm{eV}$ for $\mathrm{Eu}$, Er and Tm respectively), and hence to be stable up to temperatures greater than $1000{ }^{\circ} \mathrm{C}$ for 1 at $\%$ doping. They also possess shallow levels that may conceivably act as an excitation pathway to the $4 f$ shell. $\mathrm{RE}_{\mathrm{Ga}}-\mathrm{V}_{\mathrm{Ga}}$ pairs are more weakly bound (0.1-0.3 eV), since the RE-vacancy separation is larger; surprisingly, the binding between RE and either oxygen or native interstitials $(0.3-0.4 \mathrm{eV})$ is also quite weak; this suggests that such defects are not particularly stable against annealing. The more weakly bound structures are not expected to be present in significantly large numbers in GaN. Hence $V_{\mathrm{N}}$ is the most likely candidate for an intrinsic defect to enable $\mathrm{RE}_{\mathrm{Ga}}$ emission. In order to increase the $V_{\mathrm{N}}$ population, less N-rich conditions are required during growth. However this may have negative consequences for RE incorporation on Ga sites.

In the 5 last years our understanding of the microstructure of RE-doped III-nitrides has improved substantially; we now know that many distinct sites are optically active, and that the majority of the RE present resides in an as yet untapped reservoir in the material. Paralleling the expansion of interest in optical emission from these materials, RE-doped GaN has been proposed as suitable for high temperature spintronics applications [68]. It is an open question whether these two functionalities can be synthesized into a new form of optoelectronics, including both luminescence and spin effects. Other RE-doped systems have been demonstrated to be potential quantum computing candidates [69], and the coupling of nearby RE ions demonstrated in GaN is suggestive of another application in this field.

The authors are grateful to our colleagues in RENiBEl for their several contributions to this work, in particular K. Lorenz, B. De Vries and R.W. Martin who read and offered useful comments on parts or all of the manuscript. We thank the EU for supporting this work under Contract HRPN-CT2001-00297.

\section{References}

1. A.J. Steckl, J. Heikenfeld, D.S. Lee, M. Garter, Mat. Sci. Eng. B 81, 97 (2001)

2. T. Mukai, H. Narimatsu, S. Nakamura, Jpn J. Appl. Phys. 37, L479 (1998)

3. T. Mukai, D. Morita, S. Nakamura, J. Cryst. Growth 189, 190, 778 (1998)

4. H. Ennen, J. Schneider, G. Pomrenke, A. Axmann, Appl. Phys. Lett. 43, 943 (1983)

5. P.N. Favannec, H. L'Haridon, M. Salvi, D. Moutonnet, Y. Le Guillou, Electron. Lett. 25, 718 (1989)

6. A.J. Steckl, J.C. Heikenfeld, D.S. Lee, M.J. Garter, C.C. Baker, Y.Q. Wang, R. Jones, IEEE J. Sel. Top. Quant. 8, $749(2002)$

7. Y. Hori, T. Andreev, D. Jalabert, X. Biquard, E. Monroy, M. Tanaka, O. Oda, L.S. Dang, B. Daudin, Phys. Status Solidi B 241, 2787 (2004)

8. See http://www.renibel.net

9. K.P. O’Donnell, V. Katchkanov, K. Wang, R.W. Martin, P.R. Edwards, B. Hourahine, E. Nogales, J.F.W. Mosselmans, B. De Vries, MRS Symp. Proc. 831, E3.31 (2005)
10. J.H. Van Vleck, J. Phys. Chem. 41, 67 (1937)

11. B.R. Judd, Phys. Rev. 127, 750 (1962)

12. G.S. Ofelt, J. Chem. Phys. 37, 511 (1962)

13. C. Görller-Walrand, K. Binnemans, Handbook on the Physics and Chemistry of Rare Earths, edited by K.A. Gscheidner Jr., L. Eyring (Elsevier Science B.V., Amsterdam, 1996), Vol. 23, p. 121

14. G.H. Dieke, Spectra and Energy Levels of Rare Earth Ions in Crystals (John Wiley \& Sons Inc., New York, 1969)

15. W.T. Carnall, H. Crosswhite H.M. Crosswhite, Energy level structure and transition probabilities in the spectra of the trivalent lanthanides in LaF3, Argonne National Laboratory Report, 1977; W.T. Carnall, P.R. Fields, K. Rajnak, J. Phys. Chem. 49, 4424 (1968); W.T. Carnall, P.R. Fields, K. Rajnak, J. Phys. Chem. 4443, 4447, 4450 (1968)

16. U Vetter, Ph.D. Thesis, University of Göttingen, 2003

17. P. Goldner, Mol. Phys. 101, 903 (2003)

18. H. Bethe, Ann. Phys. Leipzig 3, 133 (1929)

19. A. Borel, L. Helm, C.A.E. Daul, Chem. Phys. Lett. 383, $584(2004)$

20. J. Wan, L. Ye, Q. Sun, X. Wang, Phys. Rev. B 5810415 (1998)

21. J. Coutinho, R. Jones, P. R. Briddon, S. Öberg, Phys. Rev. B 62, 10824 (2000)

22. J.-S. Filhol, R. Jones, M.J. Shaw, P.R. Briddon, Appl. Phys. Lett. 84, 2841 (2004)

23. V.I. Anisimov, F. Aryasetiawan, A.I. Lichtenstein, J. Phys.-Cond. Matter 9, 767 (1997)

24. Th. Frauenheim, G. Seifert, M. Elstner, Th. Niehaus, C. Köhler, M. Amkreutz, M. Sternberg, Z. Hajnal, A. Di Carlo, S. Suhai, J. Phys.-Cond. Matter 14, 3015 (2002)

25. See http://www .wien2k.at/

26. B. Hourahine, S. Sanna, B. Aradi, C. Köhler, T. Frauenheim, Physica B 376, 377, 512 (2006)

27. A. Svane, N.E. Christensen, L. Petit, Z. Szotek, W.M. Temmerman, ArXiv:cond-mat/0603288 (2006)

28. N. Rousseau, O. Briot, V. Ribes, R.L. Aulombard, MRS Symp. Proc. 764, C3.50.1 (2003); G. Halambalakis, N. Rousseau, O. Briot, S. Ruffenach, R.L. Aulombard, P.R. Edwards, K.P. O'Donnell, T. Wojtowicz, P. Ruterana, Superlattice. Microst. 36, 721 (2004)

29. T. Wojtowicz, P. Ruterana, N. Rousseau, O. Briot, S. Dalmasso, R.W. Martin, K.P. O'Donnell, Mat. Sci. Eng. B 105, 114 (2003)

30. K. Lorenz, U. Wahl, E. Alves, S. Dalmasso, R.W. Martin, K.P. O'Donnell, S. Ruffenach, O. Briot, Appl. Phys. Lett. 85, 2712 (2004); E. Nogales, R.W. Martin, K.P. O'Donnell, K. Lorenz, E. Alves, S. Ruffenach, O. Briot, Appl. Phys. Lett. 88, 031902 (2006)

31. K. Lorenz, U. Wahl, E. Alves, E. Nogales, S. Dalmasso, R.W. Martin, K.P. O'Donnell, M. Wojdak, A. Braud, T. Monteiro, T. Wojtowicz, P. Ruterana, S. Ruffenach, O. Briot, Opt. Mater. 28, 750 (2006)

32. K. Lorenz, U. Wahl, E. Alves, S. Dalmasso, R.W. Martin, K.P. O'Donnell, MRS Symp. Proc. 798, Y 5.4 (2004)

33. K. Lorenz, U. Wahl, E. Alves, T. Wojtowicz, P. Ruterana, S. Ruffenach, O. Briot, Superlattice. Microst. 36, 737 (2004)

34. S.O. Kucheyev, J.S. Williams, S.J. Pearton, Mat. Sci. Eng. R 33, 107 (2001), and references therein

35. B. Pipeleers, S.M. Hogg, A. Vantomme, J. Appl. Phys. 98, $123504(2005)$ 
36. U. Wahl, J.G. Correia, A. Czermak, S.G. Jahn, P. Jalocha, J.G. Marques, A. Rudge, F. Schopper, J.C. Soares, A. Vantomme, P. Weilhammer, Nucl. Instrum. Meth. A 524, 245 (2004)

37. B. De Vries, V. Matias, A. Vantomme, U. Wahl, E.M.C. Rita, E. Alves, A.M.L. Lopes, J.G. Correia, Appl. Phys. Lett. 84, 4304 (2004)

38. U. Wahl, E. Alves, K. Lorenz, J.G. Correia, T. Monteiro, B. De Vries, A.Vantomme, R. Vianden, Mat. Sci. Eng. B 105, 132 (2003)

39. B. de Vries, Ph.D. Thesis, University of Leuven, 2006

40. See http://www.srs.dl.ac.uk/xrs/Theory/theory.html

41. V. Katchkanov, J.F.W. Mosselmans, S. Dalmasso, K.P. O'Donnell, K. Wang, R.W. Martin, O. Briot, N. Rousseau, G. Halambalakis, MRS Symp. Proc. 798, Y5.10 (2004)

42. V. Katchkanov, J.F.W. Mosselmans, S. Dalmasso, K.P. O'Donnell, S. Hernandez, K. Wang, R.W. Martin, O. Briot, N. Rousseau, G. Halambalakis, K. Lorenz, E. Alves, Superlattice. Microst. 36, 729 (2004)

43. V. Kachkanov, Ph.D. Thesis, University of Strathclyde, unpublished

44. K.P. O’Donnell, G. Davies, J. Lumin. 26, 177 (1981)

45. V. Dierolf, C. Sandmann, J. Zavada, P. Chow, B. Hertog, J. Appl. Phys. 95, 5464 (2004)

46. S. Kim, S. Rhee, D. Turnbull, X. Li, J. Coleman, S. Bishop, P. Klein, Appl. Phys. Lett. 71, 2662 (1997)

47. F. Pellé, F. Auzel, J. Zavada, D. Lee, A. Steckl, Mat. Sci. Eng. B 105, 125 (2003)

48. B. Henderson, G.F. Imbusch, Spectroscopy of Inorganic Solids (Oxford University Press, Oxford, 2006), p. 396

49. E.E. Nyein, U. Hommerich, J. Heikenfeld, D.S. Lee, A.J. Steckl, J.M. Zavada, Appl. Phys. Lett. 82, 1655 (2003)

50. V. Katchkanov, K.P. O'Donnell, S. Dalmasso, R.W. Martin, A. Braud, Y. Nakanishi, A. Wakahara, A. Yoshida Phys. Status Solidi B 242, 1491 (2005)

51. K. Wang, R.W. Martin, K.P. O'Donnell, V. Katchkanov, E. Nogales, K. Lorenz, E. Alves, S. Ruffenach, O. Briot, Appl. Phys. Lett. 87, 112107 (2005)

52. L. Bodiou, A. Oussif, A. Braud, J.-.L Doualan, R. Moncorgé, K. Lorenz, E. Alves, Opt. Mat. 28, 780 (2006)

53. A. Braud, J.L. Doualan, R. Moncorge, B. Pipeleers, A. Vantomme, Mat. Sci. Eng. B 105, 101 (2003)

54. T. Wojtowicz, P. Ruterana, D.S. Lee, A. Steckl, Phys. Status Solidi C 2, 2484 (2005)
55. I.S. Roqan, private communication

56. M. Mamor, V. Matias, A. Vantomme, A. Colder, P. Marie, P. Ruterana, Appl. Phys. Lett. 85, 2244 (2004)

57. K. Binnemans, C. Görller-Walrand, J. Phys.-Cond. Matter 8, 1267 (1996)

58. K. Lorenz, E. Alves, U. Wahl, T. Monteiro, S. Dalmasso, R.W. Martin, K.P. O'Donnell, R. Vianden, Mat. Sci. Eng. B 105, 97 (2003)

59. K. Lorenz, U. Wahl, E.Alves, T. Wojtowicz, P. Ruterana, S. Ruffenach, O. Briot, Superlattice. Microst. 36, 737 (2004)

60. Y. Nakanishi, A. Wakahara, H. Okada, A. Yoshida, T. Ohshima, H. Itoh, T. Shibata, M. Tanaka, Phys. Status Solidi C 0, 2623 (2003)

61. R.W. Martin, private communication

62. R.W. Martin, D. Rading, R. Kersting, E. Tallarek, E. Nogales, D. Amabile, K. Wang, V. Katchkanov, C. TragerCowan, K.P. O'Donnell, I.M. Watson, V. Matias, A. Vantomme, K. Lorenz, E. Alves, Phys. Status Solidi C 3, 1927 (2006)

63. I. Roqan, K. Lorenz, K.P. O'Donnell, C. Trager-Cowan, R.W. Martin, I.M. Watson, E. Alves, Superlattice. Microst. (2006) (to be published)

64. J. Coutinho, R. Jones, M.J. Shaw, P.R. Briddon, S. Öberg, Appl. Phys. Lett. 84, 1683 (2004); J.-S. Filhol, S. Petit, R. Jones, B. Hourahine, T. Frauenheim, H. Overhof, J. Coutinho, M.J. Shaw, P.R. Briddon, MRS Symp. Proc. 798, 471 (2003)

65. S. Petit, R. Jones, M.J. Shaw, P.R. Briddon, B. Hourahine, T. Frauenheim, Phys. Rev. B 72, 073205 (2005)

66. S. Schmitt-Rink, C.M. Varma, A.F.J. Levi, Phys. Rev. Lett. 66, 2782 (1991)

67. M. Wojdak, A. Braud, J.L. Doualan, R. Moncorge, B. Pipeleers, A. Vantomme, O. Briot, Superlattice. Microst. 36, 755 (2004)

68. M. Hashimoto, S. Emura, R. Asano, H. Tanaka, N. Teraguchi, A. Suzuki, Y. Nanishi, T. Honma, N. Umesaki, H. Asahi, Phys. Status Solidi C 0, 2650 (2003); S. Dhar, O. Brandt, M. Ramsteiner, V.F. Sapega, K.H. Ploog, Phys. Rev. Lett. 94, 037205 (2005)

69. J. Wesenberg, K. Mølmer, Phys. Rev. A 68, 012320 (2003) 